\title{
A Battery Health Monitoring Framework for Planetary Rovers
}

\author{
Matthew Daigle \\ NASA Ames Research Center \\ Moffett Field, CA 94035 \\ matthew.j.daigle@nasa.gov
}

\author{
Chetan S. Kulkarni \\ SGT Inc., NASA Ames Research Center \\ Moffett Field, CA 94035 \\ chetan.s.kulkarni@nasa.gov
}

\begin{abstract}
Batteries have seen an increased use in electric ground and air vehicles for commercial, military, and space applications as the primary energy source. An important aspect of using batteries in such contexts is battery health monitoring. Batteries must be carefully monitored such that the battery health can be determined, and end of discharge and end of usable life events may be accurately predicted. For planetary rovers, battery health estimation and prediction is critical to mission planning and decision-making. We develop a model-based approach utilizing computationally efficient and accurate electrochemistry models of batteries. An unscented Kalman filter yields state estimates, which are then used to predict the future behavior of the batteries and, specifically, end of discharge. The prediction algorithm accounts for possible future power demands on the rover batteries in order to provide meaningful results and an accurate representation of prediction uncertainty. The framework is demonstrated on a set of lithium-ion batteries powering a rover at NASA Ames Research Center using real experimental field test data.
\end{abstract}

\section{TABle of Contents}

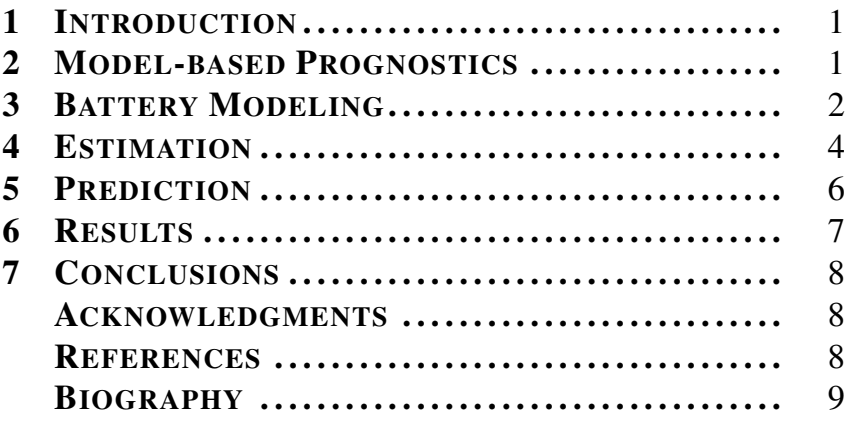

\section{INTRODUCTION}

Batteries have seen an increased use as the primary energy source in electric ground and air vehicles for commercial, military, and space applications. In such contexts, battery health monitoring (BHM) becomes a critical issue, since failure of the battery directly affects the availability of the asset. Batteries must be carefully monitored such that the battery health can be determined, and end of discharge and end of usable life events may be accurately predicted. In planetary rovers, a battery health management (BHM) system is especially important, due to its role in short- and long-term mission planning and decision-making $[1,2]$.

A BHM system consists of both battery state estimation and prediction components. While much research has been carried out on battery modeling and battery state estimation,

978-1-4799-1622-1/14/\$31.00 (C) 2014 IEEE.

1 IEEEAC Paper \#2291, Version 2, Updated 12/01/2014. the prediction component, i.e., prognostics, has only recently begun to receive attention [3-5]. Even more recently, battery prognostics has begun to be applied to electric vehicles. In $[6,7]$, end of discharge prediction was developed for an unmanned aerial vehicle platform. In [8], an approach for predicting the remaining driving range of a battery-powered car was developed. These approaches differ by the type of battery model used, and the underlying estimation and prediction algorithms. Empirical battery models are used in [6], and equivalent circuit models are used in [7-9]. Equivalent circuit models are popular because they are relatively simple and computationally efficient. For estimation, the particle filter algorithm is used in [2,3,6], whereas the unscented Kalman filter (UKF) is used in [7-9]. The UKF is generally preferred over the particle filter when the model assumptions required for the UKF can be met, due to the much higher computational requirements of the particle filter [10]. Prediction algorithms used are typically sample-based algorithms, such as Monte Carlo sampling. They differ due to how future inputs are characterized and how that uncertainty is incorporated into the predictions [11].

Our approach to a BHM system is different from previous approaches in several ways. First, we use a new electrochemistry-based battery model recently developed in [12]. Unlike similar models, it is composed only of ordinary differential equations, thus leading to a model that is as computationally efficient as equivalent circuit models. We use the UKF for state estimation, based on this new model. For prediction, we use an operator-centric approach that provides best-, average-, and worst-case usage predictions. The prognosis framework applied here is based on previous work presented in $[9,11]$.

We apply our BHM system to a planetary rover testbed developed at NASA Ames Research Center, in which we monitor a set of 24 lithium-ion batteries that are used to power the rover motors [13]. We provide experimental results validating the rover battery model, and demonstrate battery state estimation and end-of-discharge prediction using real field test data.

The paper is organized as follows. Section 2 describes the model-based prognostics framework. Section 3 summarizes the battery model. Sections 4 and 5 discuss the estimation and prediction approaches, respectively. Section 6 presents the experimental results. Section 7 concludes the paper.

\section{Model-based Prognostics}

In this section, we first formulate the prognostics problem, and present the uncertainty representation framework [11] We then provide an architecture for model-based prognostics that will be applied for BHM. 
Prognostics

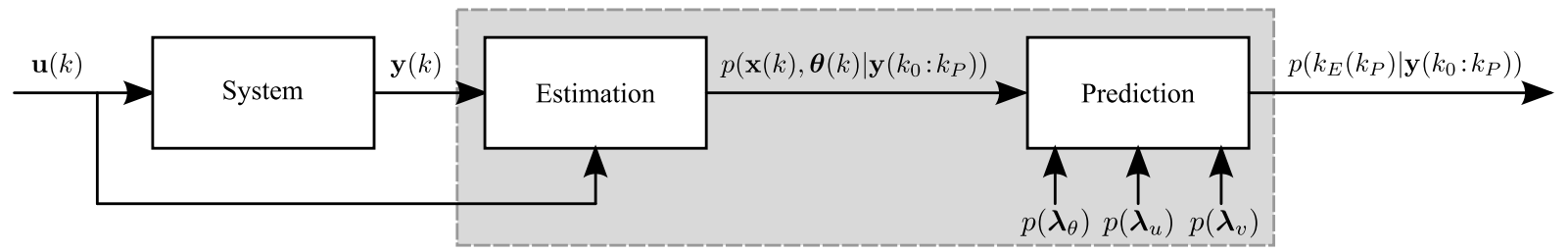

Figure 1. Prognostics architecture.

\section{Problem Formulation}

We assume the system model may be generally defined as

$$
\begin{aligned}
\mathbf{x}(k+1) & =\mathbf{f}(k, \mathbf{x}(k), \boldsymbol{\theta}(k), \mathbf{u}(k), \mathbf{v}(k)), \\
\mathbf{y}(k) & =\mathbf{h}(k, \mathbf{x}(k), \boldsymbol{\theta}(k), \mathbf{u}(k), \mathbf{n}(k)),
\end{aligned}
$$

where $k$ is the discrete time variable, $\mathbf{x}(k) \in \mathbb{R}^{n_{x}}$ is the state vector, $\boldsymbol{\theta}(k) \in \mathbb{R}^{n_{\theta}}$ is the unknown parameter vector, $\mathbf{u}(k) \in \mathbb{R}^{n_{u}}$ is the input vector, $\mathbf{v}(k) \in \mathbb{R}^{n_{v}}$ is the process noise vector, $\mathbf{f}$ is the state equation, $\mathbf{y}(k) \in \mathbb{R}^{n_{y}}$ is the output vector, $\mathbf{n}(k) \in \mathbb{R}^{n_{n}}$ is the measurement noise vector, and $\mathbf{h}$ is the output equation. ${ }^{2}$ The unknown parameter vector $\boldsymbol{\theta}(k)$ is used to capture explicit model parameters whose values are unknown and time-varying stochastically.

Prognostics is concerned with predicting the occurrence of some event $E$ that is defined with respect to the states, parameters, and inputs of the system. We define the event as the earliest instant that some event threshold $T_{E}: \mathbb{R}^{n_{x}} \times$ $\mathbb{R}^{n_{\theta}} \times \mathbb{R}^{n_{u}} \rightarrow \mathbb{B}$, where $\mathbb{B} \triangleq\{0,1\}$ changes from the value 0 to 1 . That is, the time of the event $k_{E}$ at some time of prediction $k_{P}$ is defined as

$$
k_{E}\left(k_{P}\right) \triangleq \inf \left\{k \in \mathbb{N}: k \geq k_{P} \wedge T_{E}(\mathbf{x}(k), \boldsymbol{\theta}(k), \mathbf{u}(k))=1\right\} .
$$

The time remaining until that event, $\Delta k_{E}$, is defined as

$$
\Delta k_{E}\left(k_{P}\right) \triangleq k_{E}\left(k_{P}\right)-k_{P}
$$

For batteries, the event $E$ corresponds to end of discharge (EOD). EOD is defined by a voltage threshold $V_{E O D}$, where $T_{E}$ is defined by $V<V_{E O D}$. When the battery voltage is less than the cutoff voltage, $V_{E O D}$, EOD is reached and $T_{E}$ evaluates to 1 .

The prognostics problem is inherently uncertain, due to the random nature of the system evolution (due to $\mathbf{v}(k)$ ), and nondeterministic future inputs $\left(\mathbf{u}(k)\right.$ for $\left.k>k_{P}\right)$. Therefore, $k_{E}$ and $\Delta k_{E}$ are random variables, and we must compute $p\left(k_{E}\left(k_{P}\right) \mid \mathbf{y}\left(k_{0}: k_{P}\right)\right)$ and/or $p\left(\Delta k_{E}\left(k_{P}\right) \mid \mathbf{y}\left(k_{0}: k_{P}\right)\right)[9,14]$.

\section{Uncertainty Representation}

In order to predict $k_{E}$, four sources of uncertainty must be dealt with in general [11]: $(i)$ the initial state at time $k_{P}$, $\mathbf{x}\left(k_{p}\right)$; (ii) the parameter values $\boldsymbol{\theta}(k)$ for all $k \geq k_{P}$, denoted as $\boldsymbol{\Theta}_{k_{P}}$ (the subscript $k_{P}$ indicates the start time of the trajectory); (iii) the inputs $\mathbf{u}(k)$ for all $k \geq k_{P}$, denoted as $\mathbf{U}_{k_{P}}$; and (iv) the process noise $\mathbf{v}(k)$ for all $k \geq k_{P}$, denoted as $\mathbf{V}_{k_{P}}$. In order to make a prediction that accounts for this uncertainty, we require the probability distributions $p(\mathbf{x}), p\left(\boldsymbol{\Theta}_{k_{P}}\right), p\left(\mathbf{U}_{k_{P}}\right)$, and $p\left(\mathbf{V}_{k_{P}}\right)$.

\footnotetext{
${ }^{2}$ Bold typeface denotes vectors, and $n_{a}$ denotes the length of a vector $\mathbf{a}$.
}

For describing the probability distribution of a generic trajectory $\mathbf{A}_{k}$, we introduce a set of surrogate random variables $\boldsymbol{\lambda}_{a}=\left[\lambda_{a}^{1} \lambda_{a}^{2} \ldots\right]$ [11]. We describe a trajectory using $\boldsymbol{\lambda}_{a}$ and instead define $p\left(\boldsymbol{\lambda}_{a}\right)$, which in turn defines $p\left(\mathbf{A}_{k}\right)$. These surrogate variables can be used to describe trajectories in myriad ways. For the parameter, input, and process noise trajectories we have the surrogate variables $\boldsymbol{\lambda}_{\theta}, \boldsymbol{\lambda}_{u}$, and $\boldsymbol{\lambda}_{v}$. Additional discussion on the use of surrogate variables can be found in [11].

\section{Prognostics Architecture}

We adopt a model-based prognostics architecture [11, 15], in which there are two sequential problems, (i) the estimation problem, which requires determining a joint stateparameter estimate $p\left(\mathbf{x}(k), \boldsymbol{\theta}(k) \mid \mathbf{y}\left(k_{0}: k_{P}\right)\right)$ based on the history of observations up to time $k, \mathbf{y}\left(k_{0}: k_{P}\right)$, and (ii) the prediction problem, which determines at $k_{P}$, using $p\left(\mathbf{x}(k), \boldsymbol{\theta}(k) \mid \mathbf{y}\left(k_{0}: k_{P}\right)\right), p\left(\boldsymbol{\lambda}_{\theta}\right), p\left(\boldsymbol{\lambda}_{u}\right)$, and $p\left(\boldsymbol{\lambda}_{v}\right)$, a probability distribution $p\left(k_{E}\left(k_{P}\right) \mid \mathbf{y}\left(k_{0}: k_{P}\right)\right)$. The distribution for $\Delta k_{E}$ can be trivially computed from $p\left(k_{E}\left(k_{P}\right) \mid \mathbf{y}\left(k_{0}: k_{P}\right)\right)$ by subtracting $k_{P}$ from $k_{E}\left(k_{P}\right)$.

The prognostics architecture is shown in Fig. 1 [11]. In discrete time $k$, the system is provided with inputs $\mathbf{u}_{k}$ and provides measured outputs $\mathbf{y}_{k}$. The estimation module uses this information, along with the system model, to compute an estimate $p\left(\mathbf{x}(k), \boldsymbol{\theta}(k) \mid \mathbf{y}\left(k_{0}: k\right)\right)$. The prediction module uses the joint state-parameter distribution and the system model, along with the distributions for the surrogate variables, $p\left(\boldsymbol{\lambda}_{\theta}\right)$, $p\left(\boldsymbol{\lambda}_{u}\right)$, and $p\left(\boldsymbol{\lambda}_{v}\right)$, to compute the probability distribution $p\left(k_{E}\left(k_{P}\right) \mid \mathbf{y}\left(k_{0}: k_{P}\right)\right)$ at given prediction times $k_{P}$.

\section{BATTERY MODELing}

In order to apply model-based prognostics, we require a model. For this purpose, we employ an electrochemistrybased lithium ion battery model developed in [12]. In contrast to the empirical and equivalent circuit battery models used in previous approaches [3,8,9], the new model presented in [12] is based on the underlying electrochemical equations, but at a level of abstraction high enough that the model is still efficient with the improved fidelity. The model is represented as a set of ordinary differential equations and can be converted to a discrete-time representation and solved efficiently with a sample time of $1 \mathrm{~s}$. We summarize here the model equations and refer the reader to [12] for additional details.

The battery model computes the voltage as a function of time given the current drawn from the battery. Several electrochemical processes contribute to the cell's potential. The different potentials are summarized in Fig. 2 (adapted from [16]). The overall battery voltage $V(t)$ is the difference between the potential at the positive current collector, $\phi_{s}(0, t)$, and the negative current collector, $\phi_{s}(L, t)$, minus resistance losses at the current collectors (not shown in the 


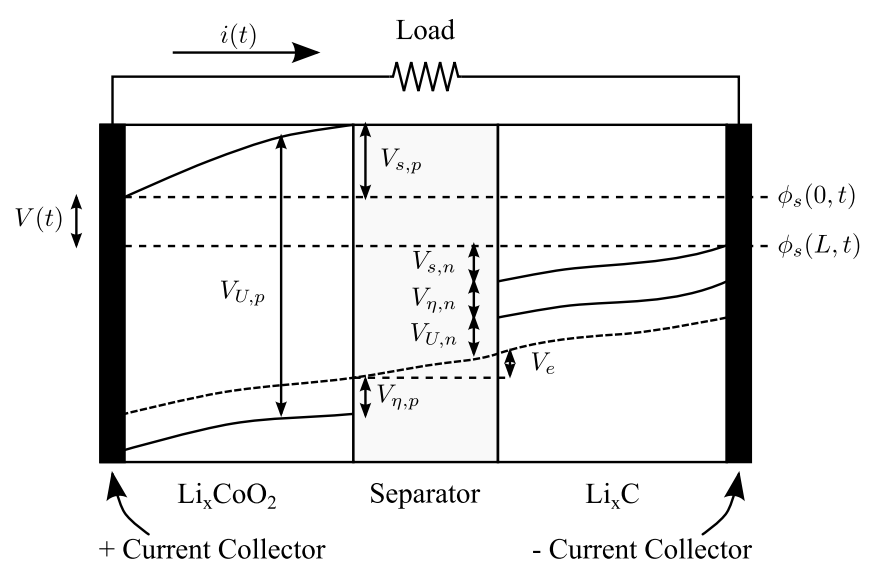

Figure 2. Battery voltages.

diagram).

The potentials at the current collectors are described by several voltage terms. At the positive current collector is the equilibrium potential $V_{U, p}$. This voltage is then reduced by $V_{s, p}$, due to the solid-phase ohmic resistance, and $V_{\eta, p}$, the surface overpotential. The electrolyte ohmic resistance then causes another drop $V_{e}$. At the negative electrode, there is a drop $V_{\eta, n}$ due to the surface overpotential, and a drop $V_{s, n}$ due to the solid-phase resistance. The voltage drops again due to the equilibrium potential at the negative current collector $V_{U, n}$. We describe each of these voltages in the following subsections.

\section{Equilibrium Potential}

The equilibrium potential (also known as the open-circuit voltage) is captured using the Nernst equation:

$$
V_{U, i}=U_{0}+\frac{R T}{n F} \ln \left(\frac{1-x_{i}}{x_{i}}\right)+V_{\mathrm{INT}, i},
$$

where $i$ refers to the electrode ( $n$ for negative or $p$ for positive), $U_{0}$ is a reference potential, $R$ is the universal gas constant, $T$ is the electrode temperature (in $\mathrm{K}$ ), $n$ is the number of electrons transferred in the reaction $(n=1$ for Liion), $F$ is Faraday's constant, $x$ is the mole fraction of lithium ions in the lithium-intercalated host material [17]. During discharge, $\mathrm{Li}$ ions move out of the negative electrode and into the positive electrode, so $x_{n}$ decreases while $x_{p}$ increases, and $V_{U, p}-V_{U, n}$ will decrease.

Here, $V_{\mathrm{INT}, i}$ is the activity correction term ( 0 in the ideal condition). We use the Redlich-Kister expansion:

$V_{\mathrm{INT}, i}=\frac{1}{n F}\left(\sum_{k=0}^{N_{i}} A_{i, k}\left(\left(2 x_{i}-1\right)^{k+1}-\frac{2 x_{i} k\left(1-x_{i}\right)}{\left(2 x_{i}-1\right)^{1-k}}\right)\right)$.

Here we use $N_{p}=12$ and $N_{n}=0$. The identified parameters are given in Table 1 [12].

We let $q_{i}$ represent the amount of $\mathrm{Li}$ ions in electrode $i$, as measured in Coulombs. The flow of Li ions is opposite to the flow of current, so $q_{i}$ changes in the same direction as $x_{i}$, and we have:

$$
x_{i}=\frac{q_{i}}{q^{\max }}
$$

Table 1. Battery Model Parameters

\begin{tabular}{cr}
\hline Parameter & Value \\
\hline$U_{0, p}$ & $4.03 \mathrm{~V}$ \\
$A_{p, 0}$ & $-33642.23 \mathrm{~J} / \mathrm{mol}$ \\
$A_{p, 1}$ & $0.11 \mathrm{~J} / \mathrm{mol}$ \\
$A_{p, 2}$ & $23506.89 \mathrm{~J} / \mathrm{mol}$ \\
$A_{p, 3}$ & $-74679.26 \mathrm{~J} / \mathrm{mol}$ \\
$A_{p, 4}$ & $14359.34 \mathrm{~J} / \mathrm{mol}$ \\
$A_{p, 5}$ & $307849.79 \mathrm{~J} / \mathrm{mol}$ \\
$A_{p, 6}$ & $85053.13 \mathrm{~J} / \mathrm{mol}$ \\
$A_{p, 7}$ & $-1075148.06 \mathrm{~J} / \mathrm{mol}$ \\
$A_{p, 8}$ & $2173.62 \mathrm{~J} / \mathrm{mol}$ \\
$A_{p, 9}$ & $991586.68 \mathrm{~J} / \mathrm{mol}$ \\
$A_{p, 10}$ & $283423.47 \mathrm{~J} / \mathrm{mol}$ \\
$A_{p, 11}$ & $-163020.34 \mathrm{~J} / \mathrm{mol}$ \\
$A_{p, 12}$ & $-470297.35 \mathrm{~J} / \mathrm{mol}$ \\
\hline$U_{0, n}$ & $0.01 \mathrm{~V}$ \\
$A_{n, 0}$ & $86.19 \mathrm{~J} / \mathrm{mol}$ \\
\hline
\end{tabular}

where $q^{\max }=q_{p}+q_{n}$ refers to the total amount of available Li ions. It follows that $x_{p}+x_{n}=1$. When fully charged, $x_{p}=0.4$ and $x_{n}=0.6$. When fully discharged, $x_{p}=1$ and $x_{n}=0 .^{3}$

\section{Concentration Overpotential}

When a battery is discharged, the reactions take place at the surface of the electrode and this results in a concentration gradient across the cell. The model accommodates this by splitting the total electrode volume into two individual control volumes (CVs), one for the bulk (with subscript $b$ ) and one for the surface (with subscript $s$ ).

For the volumes, the concentration of Li ions is computed as

$$
\begin{aligned}
c_{b, i} & =\frac{q_{b, i}}{v_{b, i}} \\
c_{s, i} & =\frac{q_{s, i}}{v_{s, i}},
\end{aligned}
$$

where, for CV $v$ in electrode $i, c_{v, i}$ is the concentration and $v_{v, i}$ is the volume. We define $v_{i}=v_{b, i}+v_{s, i}$. Note now that the following relations hold:

$$
\begin{aligned}
q_{p} & =q_{s, p}+q_{b, p} \\
q_{n} & =q_{s, n}+q_{b, n} \\
q^{\max } & =q_{s, p}+q_{b, p}+q_{s, n}+q_{b, n} .
\end{aligned}
$$

As the battery discharges, Li ions move from the surface layer at the negative electrode, through the bulk, and to the surface layer at the positive electrode, in order to match the flow of electrons. Li ions also move from the bulk CV to the surface due to the concentration gradient. The diffusion rate from the bulk to the surface is expressed as

$$
\dot{q}_{b s, i}=\frac{1}{D}\left(c_{b, i}-c_{s, i}\right),
$$

where $D$ is the diffusion constant.

\footnotetext{
${ }^{3}$ For $\mathrm{Li}_{x} \mathrm{CoO}_{2}, x$ must be at least 0.4 ; $\mathrm{Li}$ cannot be reversibly removed
} beyond that [17]. 
The $q$ variables are described as

$$
\begin{aligned}
& \dot{q}_{s, p}=i_{a p p}+\dot{q}_{b s, p} \\
& \dot{q}_{b, p}=-\dot{q}_{b s, p}+i_{a p p}-i_{a p p} \\
& \dot{q}_{b, n}=-\dot{q}_{b s, n}+i_{a p p}-i_{a p p} \\
& \dot{q}_{s, n}=-i_{a p p}+\dot{q}_{b s, n},
\end{aligned}
$$

where $i_{a p p}$ is the applied electric current. Initially, $c_{s, i}=c_{b, i}$ thus diffusion is zero.

The concentration overpotential is the difference in voltage between the two CVs due to the difference in concentration. Using the expression for equilibrium potential, we can compute the potential for the bulk volume and the potential for the surface layer; the difference between them is the concentration overpotential. So, we can explicitly account for the concentration overpotential simply by using as the expression for equilibrium potential, the equilibrium potential of the surface layer [12], i.e.,

$$
V_{U, i}=U_{0}+\frac{R T}{n F} \ln \left(\frac{1-x_{s, i}}{x_{s, i}}\right)+V_{\mathrm{INT}, i}
$$

where $x_{s, i}$ is computed using

$$
x_{s, i}=\frac{q_{s, i}}{q_{s, i}^{\max }}
$$

and

$$
q_{s, i}^{\max }=q^{\max } \frac{v_{s, i}}{v_{i}}
$$

\section{Ohmic Overpotential}

The voltage drops due to the solid-phase ohmic resistances, the electrolyte ohmic resistance, and the resistances at the current collectors are constant and lumped together into resistance $R_{o}$ :

$$
V_{o}=i_{a p p} R_{o}
$$

\section{Surface Overpotential}

The overpotentials due to charge transfer resistance and SEI kinetics are described by the Butler-Volmer equation, which, for $\mathrm{Li}$ ion batteries, reduces to

$$
V_{\eta, i}=\frac{R T}{F \alpha} \operatorname{arcsinh}\left(\frac{J_{i}}{2 J_{i 0}}\right)
$$

where $J_{i}$ is the current density, and $J_{i 0}$ is the exchange current density. The current densities are defined as

$$
\begin{aligned}
J_{i} & =\frac{i}{S_{i}} \\
J_{i 0} & =k_{i}\left(1-x_{s, i}\right)^{\alpha}\left(x_{s, i}\right)^{1-\alpha},
\end{aligned}
$$

where $k_{i}$ is a lumped parameter of several constants including a rate coefficient, electrolyte concentration, and maximum ion concentration.

\section{State of Charge}

The state of charge (SOC) of a battery is defined to be 1 when the battery is fully charged and 0 when the battery is fully discharged by convention. In this model, it is analogous to the mole fraction $x_{n}$, but scaled from 0 to 1 . We distinguish here between nominal SOC and apparent SOC [12]. Nominal SOC is computed based on the combination of the bulk and surface layer CVs in the negative electrode, whereas apparent SOC is be computed based only on the surface layer. When a battery reaches the voltage cutoff, apparent SOC is 0 , and nominal SOC is greater than 0 (how much greater depends on the difference between the diffusion rate and the current drawn). Once the concentration gradient settles out, the surface layer will be partially replenished and apparent SOC will rise while nominal SOC remains the same. Nominal $(n)$ and apparent (a) SOC are defined using

$$
\begin{aligned}
S O C_{n} & =\frac{q_{n}}{0.6 q^{\max }} \\
S O C_{a} & =\frac{q_{s, n}}{0.6 q^{\max _{s, n}}},
\end{aligned}
$$

where $q^{\max _{s, n}}=q^{\max } \frac{v_{s, n}}{v_{n}} .4$

\section{Battery Voltage}

Battery voltage can now be expressed as follows:

$$
V=V_{U, p}-V_{U, n}-V_{o}^{\prime}-V_{\eta, p}^{\prime}-V_{\eta, n}^{\prime},
$$

where

$$
\begin{aligned}
\dot{V}_{o}^{\prime} & =\left(V_{o}-V_{o}^{\prime}\right) / \tau_{o} \\
\dot{V}_{\eta, p}^{\prime} & =\left(V_{\eta, p}-V_{\eta, p}^{\prime}\right) / \tau_{\eta, p} \\
\dot{V}_{\eta, n}^{\prime} & =\left(V_{\eta, n}-V_{\eta, n}^{\prime}\right) / \tau_{\eta, n},
\end{aligned}
$$

and the $\tau$ parameters are empirical time constants (used since the voltages do not change instantaneously).

The model contains as states $\mathbf{x}, q_{s, p}, q_{b, p}, q_{b, n}, q_{s, n}, V_{o}^{\prime}, V_{\eta, p}^{\prime}$, and $V_{\eta, n}^{\prime}$. The single model output is $V$. Identified model parameters are given in Table 2 [12].

\section{Model Validation}

Measured and predicted voltage curves for a 0.044 A discharge (approximately equal to open-circuit voltage) are shown in Fig. 3, and for a 2 A discharge in Fig. 4. The data here was obtained from a laboratory setting using the rover batteries. Clearly, the model is very accurate. Measured and predicted voltage curves for battery data from a rover field test are shown in Fig. 5. The model is still quite accurate in this case. Note that the spike in predicted voltage occurring near $4000 \mathrm{~s}$ is due to a gap in the recorded data, causing an incorrect sample time to be given to the model.

\section{ESTIMATION}

In order to accurately predict the future behavior of the battery, we must first estimate its state. For this purpose, we use the unscented Kalman filter (UKF) [18, 19]. Among nonlinear filters, the UKF generally has better accuracy than

${ }^{4}$ Note that SOC of 1 corresponds to the point where $q_{n}=0.6 q^{\max _{s, n}}$, since
the mole fraction at the positive electrode cannot go below 0.4 , as described earlier. 
Table 2. Battery Model Parameters

\begin{tabular}{cc}
\hline Parameter & Value \\
\hline$q^{\max }$ & $1.32 \times 10^{4} \mathrm{C}$ \\
$R$ & $8.314 \mathrm{~J} / \mathrm{mol} / \mathrm{K}$ \\
$T$ & $292 \mathrm{~K}$ \\
$F$ & $96487 \mathrm{C} / \mathrm{mol}$ \\
$n$ & 1 \\
$D$ & $7.0 \times 10^{6} \mathrm{~mol} \mathrm{~s} / \mathrm{C} / \mathrm{m}^{3}$ \\
$\tau_{o}$ & $10 \mathrm{~s}$ \\
$\alpha$ & 0.5 \\
$R_{o}$ & $0.085 \Omega$ \\
\hline$S_{p}$ & $2 \times 10^{-4} \mathrm{~m}^{2}$ \\
$k_{p}$ & $2 \times 10^{4} \mathrm{~A} / \mathrm{m}^{2}$ \\
$v_{s, p}$ & $2 \times 10^{-6} \mathrm{~m}^{3}$ \\
$v_{b, p}$ & $2 \times 10^{-5} \mathrm{~m}^{3}$ \\
$\tau_{\eta, p}$ & $90 \mathrm{~s}$ \\
\hline$S_{n}$ & $2 \times 10^{-4} \mathrm{~m}^{2}$ \\
$k_{n}$ & $2 \times 10^{4} \mathrm{~A} / \mathrm{m}^{2}$ \\
$v_{s, n}$ & $2 \times 10^{-6} \mathrm{~m}^{3}$ \\
$v_{b, n}$ & $2 \times 10^{-5} \mathrm{~m}^{3}$ \\
$\tau_{\eta, n}$ & $90 \mathrm{~s}$ \\
\hline
\end{tabular}



Figure 3. Comparison of predicted and measured opencircuit voltage.

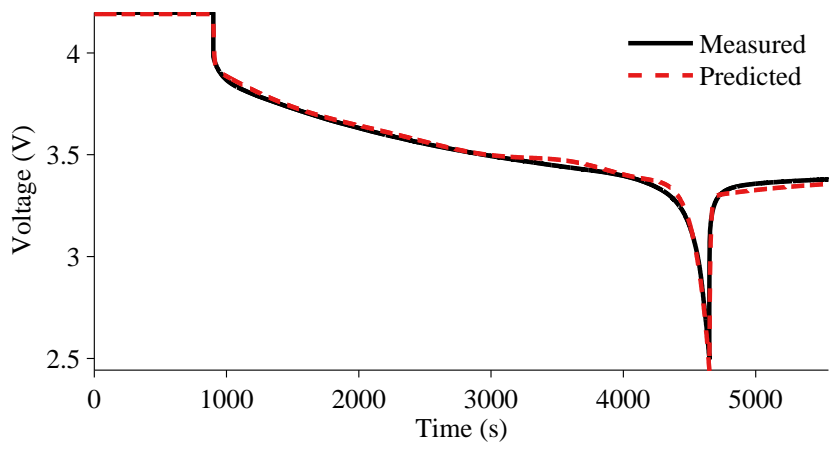

Figure 4. Comparison of predicted and measured voltage for a constant 2 A discharge.

the extended Kalman filter, and avoids the high computational cost of particle filters [20]. We summarize the filter basics here; more details may be found in $[18,19]$.

The UKF approximates a distribution using the unscented transform (UT). The UT takes a random variable $\mathbf{x} \in \mathbb{R}^{\mathbf{n}_{\mathbf{x}}}$, with mean $\overline{\mathbf{x}}$ and covariance $\mathbf{P}_{\mathbf{x x}}$, which is related to a second

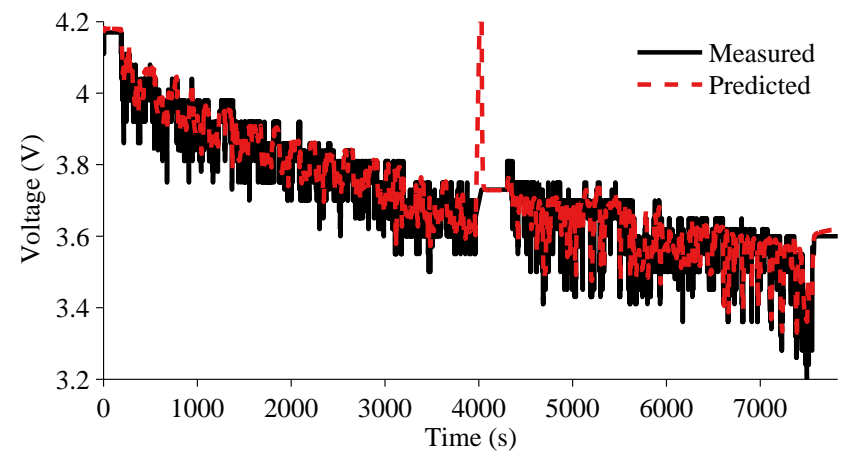

Figure 5. Comparison of predicted and measured voltage for a rover battery during a field test.

random variable $\mathbf{y}$ by some nonlinear function $\mathbf{y}=\mathbf{g}(\mathbf{x})$, and computes the mean $\overline{\mathbf{y}}$ and covariance $\mathbf{P}_{\mathbf{y y}}$ using a set of deterministically selected weighted samples, called sigma points [18]. $\quad \mathcal{X}^{i}$ denotes the $i$ th sigma point from $\mathbf{x}$ and $w^{i}$ denotes its weight. The sigma points are always chosen such that the mean and covariance match those of the original distribution, $\overline{\mathbf{x}}$ and $\mathbf{P}_{\mathbf{x x}}$. Each sigma point is passed through $\mathrm{g}$ to obtain new sigma points $\mathcal{Y}$, i.e.,

$$
\mathcal{Y}^{i}=\mathbf{g}\left(\mathcal{X}^{\mathbf{i}}\right)
$$

with mean and covariance

$$
\begin{aligned}
\overline{\mathbf{y}} & =\sum_{i} w^{i} \mathcal{Y}^{i} \\
\mathbf{P}_{y y} & =\sum_{i} w^{i}\left(\mathcal{Y}^{i}-\overline{\mathbf{y}}\right)\left(\mathcal{Y}^{i}-\overline{\mathbf{y}}\right)^{T} .
\end{aligned}
$$

We use the symmetric unscented transform, which selects $2 n_{x}+1$ sigma points symmetrically about the mean [19]:

$$
\begin{aligned}
w^{i}= & \begin{cases}\frac{\kappa}{\left(n_{x}+\kappa\right)}, & i=0 \\
\frac{1}{2\left(n_{x}+\kappa\right)}, & i=1, \ldots, 2 n_{x}\end{cases} \\
\boldsymbol{\mathcal { X }}^{i}= & \begin{array}{ll}
\overline{\mathbf{x}}, & i=0 \\
\overline{\mathbf{x}}+\left(\sqrt{\left(n_{x}+\kappa\right) \mathbf{P}_{x x}}\right)^{i}, & i=1, \ldots, n_{x} \\
\overline{\mathbf{x}}-\left(\sqrt{\left(n_{x}+\kappa\right) \mathbf{P}_{x x}}\right)^{i}, i=n_{x}+1, \ldots, 2 n_{x}
\end{array}
\end{aligned}
$$

where $\left(\sqrt{\left(n_{x}+\kappa\right) \mathbf{P}_{x x}}\right)^{i}$ refers to the $i$ th column of the matrix square root of $\left(n_{x}+\kappa\right) \mathbf{P}_{x x}$ (e.g., computed using the Cholesky decomposition). The number $\kappa$ is a free parameter that can be used to tune the higher order moments of the distribution, and if $\mathbf{x}$ is assumed Gaussian, then selecting $\kappa=3-n_{x}$ is recommended [18].

The UKF assumes the general nonlinear form of the state and output equations, but is restricted to additive Gaussian noise. First, $n_{s}$ sigma points $\hat{\mathcal{X}}_{k-1 \mid k-1}$ are derived from the current mean $\hat{\mathbf{x}}_{k-1 \mid k-1}$ and covariance estimates $\mathbf{P}_{k-1 \mid k-1}$. 
The prediction step is:

$$
\begin{aligned}
& \hat{\boldsymbol{X}}_{k \mid k-1}^{i}=\mathbf{f}\left(\hat{\boldsymbol{\mathcal { X }}}_{k-1 \mid k-1}^{i}, \mathbf{u}_{k-1}\right), i=1, \ldots, n_{s} \\
& \hat{\mathcal{Y}}_{k \mid k-1}^{i}=\mathbf{h}\left(\hat{\boldsymbol{\mathcal { X }}}_{k \mid k-1}^{i}\right), i=1, \ldots, n_{s} \\
& \hat{\mathbf{x}}_{k \mid k-1}=\sum_{i}^{n_{s}} w^{i} \boldsymbol{\mathcal { X }}_{k \mid k-1}^{i} \\
& \hat{\mathbf{y}}_{k \mid k-1}=\sum_{i}^{n_{s}} w^{i} \mathcal{Y}_{k \mid k-1}^{i} \\
& \mathbf{P}_{\mathbf{k} \mid \mathbf{k}-\mathbf{1}}=\mathbf{Q}+ \\
& \sum_{i}^{n_{s}} w^{i}\left(\boldsymbol{\mathcal { X }}_{k \mid k-1}^{i}-\hat{\mathbf{x}}_{k \mid k-1}\right)\left(\mathcal{X}_{k \mid k-1}^{i}-\hat{\mathbf{x}}_{k \mid k-1}\right)^{T},
\end{aligned}
$$

where $\mathbf{Q}$ is the process noise covariance matrix.

The update step is:

$$
\mathbf{P}_{y y}=\mathbf{R}+\sum_{i}^{n_{s}} w^{i}\left(\mathcal{Y}_{k \mid k-1}^{i}-\hat{\mathbf{y}}_{k \mid k-1}\right)\left(\mathcal{Y}_{k \mid k-1}^{i}-\hat{\mathbf{y}}_{k \mid k-1}\right)^{T}
$$$$
\mathbf{P}_{x y}=\sum_{i}^{n_{s}} w^{i}\left(\boldsymbol{\mathcal { X }}_{k \mid k-1}^{i}-\hat{\mathbf{x}}_{k \mid k-1}\right)\left(\mathcal{Y}_{k \mid k-1}^{i}-\hat{\mathbf{y}}_{k \mid k-1}\right)^{T}
$$

$$
\begin{aligned}
\mathbf{K}_{k} & =\mathbf{P}_{x y} \mathbf{P}_{y y}^{-1} \\
\hat{\mathbf{x}}_{k \mid k} & =\hat{\mathbf{x}}_{k \mid k-1}+\mathbf{K}_{k}\left(\mathbf{y}_{k}-\hat{\mathbf{y}}_{k \mid k-1}\right) \\
\mathbf{P}_{k \mid k} & =\mathbf{P}_{k \mid k-1}-\mathbf{K}_{k} \mathbf{P}_{y y} \mathbf{K}_{k}^{T},
\end{aligned}
$$

where $\mathbf{R}$ is the sensor noise covariance matrix.

For the battery, we have 7 states, resulting in 15 sigma points. An estimate of SOC can be computed from the estimates of the states. This can be computed using the unscented transform, in which the sigma points for the state are transformed into sigma points for SOC, using the equations for computing SOC from the battery charge. We can then extract mean and variance of the SOC estimate from these transformed sigma points.

\section{Prediction}

Prediction is initiated at a given time $k_{P}$ using the current joint state-parameter estimate, $p\left(\mathbf{x}\left(k_{P}\right), \boldsymbol{\theta}\left(k_{P}\right) \mid \mathbf{y}\left(k_{0}: k_{P}\right)\right)$. The goal is to compute $p\left(k_{E}\left(k_{P}\right) \mid \mathbf{y}\left(k_{0}: k_{P}\right)\right)$ using the stateparameter estimates and assumptions about uncertainty regarding the future parameter, input, and process noise values.

In this work, we assume all parameters are known, so $\boldsymbol{\theta}(k)$ is empty. Further, we assume that, because the model is so accurate, process noise is negligible. Of course, process noise is not zero, but in this case the uncertainty in the future inputs dominates significantly, so including process noise has virtually no effect on the prediction results. In the following, we describe the approach for the general case as originally developed in [11].

For one realization of each of the uncertain quantities at prediction time $k_{P}$ : the state $\mathbf{x}\left(k_{P}\right)$, the parameter trajectory

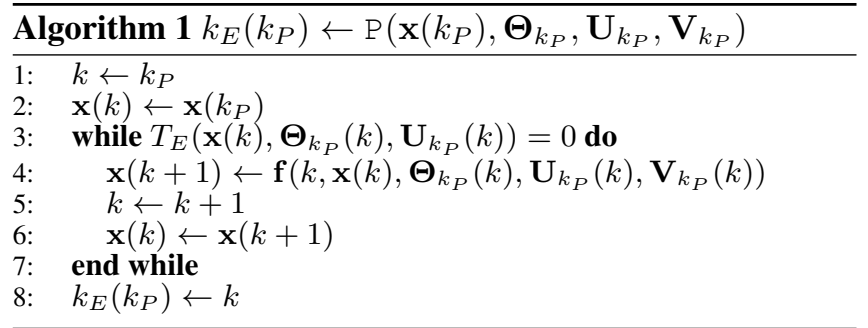

$\Theta_{k_{P}}$, the input trajectory $\mathbf{U}_{k_{P}}$, and the process noise trajectory $\mathbf{V}_{k_{P}}$, the corresponding realization of $k_{E}$ can be computed with the system model as shown in Algorithm 1 [11]. In Algorithm 1, the function P simulates the system model until the threshold $T_{E}$ evaluates to 1 .

This algorithm requires computing first realizations of the state-parameter distribution, the parameter trajectory, the input trajectory, and the process noise trajectory. The distribution for the state comes from the UKF, and the distributions for the parameter, input, and process noise trajectories are defined indirectly by the set of surrogate variables. So, we are interested in computing the distribution for $k_{E}$ from the distributions for $p\left(\mathbf{x}\left(k_{P}\right), \boldsymbol{\theta}\left(k_{P}\right)\right), p\left(\boldsymbol{\lambda}_{\theta}\right), p\left(\boldsymbol{\lambda}_{u}\right)$, and $p\left(\boldsymbol{\lambda}_{v}\right)$ (see Fig. 1).

In this paper, since only future input uncertainty is considered, we need to define only the surrogate variables for the future input trajectory. Here, future input trajectories may take many complex forms. Instead of representing these complexities directly, we consider instead an equivalent constant-loading distribution for the future inputs. That is, we assume that the future power usage for a battery will be constant with the value drawn from some distribution.

Prediction methods differ by how they sample from the given distributions, and how they call P. Different methods were investigated in [11], including Monte Carlo sampling, unscented transform sampling, and the inverse first-orderreliability method (FORM). In the case of the rover, the operator really only needs to know EOD predictions for best, average-, and worst-case usage scenarios. For the state estimate, we use as samples the sigma points provided by the UKF. Each sample is simulated forward three times, once for each use case. From this we obtain best-, average-, and worstcase EOD predictions, each with some small variance (due to the state estimate variance).

If instead the operator desires a probability distribution, then an equivalent constant-loading distribution can be selected. We can determine the appropriate statistics for an equivalent constant-loading distribution by analyzing field experiments. Assuming a normal distribution, we can compute the average power for each field experiment, then compute the mean and variance of the average power. For a particular realization of the input, we can sample the average power from this distribution, and use that power for $k \geq k_{P}$. In this paper we use a mean power draw of $3.3 \mathrm{~W}$ with a variance of 1.0 .

In this case, we have one surrogate variable needed to describe the future input, which is used to define the constant power draw, as defined by the determined statistics. In order to sample efficiently from this distribution, we use the unscented transform method, as originally described in [9]. The unscented transform described in Section 4 is used to sample from the future input distribution, and in this case only 3 samples are needed since there is only a single 


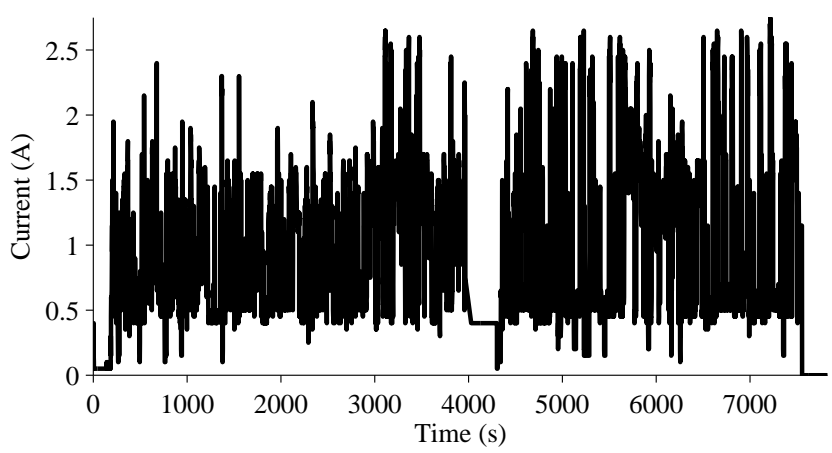

Figure 6. Battery current.

surrogate variable. This is much more efficient than the hundreds or thousands of samples required for Monte Carlo sampling. Prediction then proceeds in the normal way, with the $\mathrm{P}$ function being called for the sigma point combinations (each state sigma point is simulated for each future input sigma point). Using the unscented transform equations we can then determine the statistics of EOD, namely, the mean and variance. (For additional statistical moments, extended versions of the unscented transform or Monte Carlo sampling are required. Alternatively, the inverse FORM method can be used to determine cumulative distribution function values at desired points.) Mathematical details of this approach are provided in $[9,11]$.

\section{RESULTS}

In this section, we present experimental results obtained from the planetary rover testbed at NASA Ames Research Center [13]. The rover motors are powered by 24 lithium-ion batteries, with two strings of 12 batteries in series, connected in parallel. So, each battery sees only about half of the total current required to operate the motors. Fully charged, each battery supplies $4.2 \mathrm{~V}$. The batteries can be safely discharged to $2.5 \mathrm{~V}$, but the drop from about $3.3 \mathrm{~V}$ down to $2.5 \mathrm{~V}$ happens relatively quickly, so to prevent overdischarge, the voltage cutoff used is $V_{E O D}=3.3 \mathrm{~V}$.

The BHM system monitors the health state of each battery, and periodically provides EOD predictions, to let the rover operator know how much longer the rover can be used in the field. We demonstrate the BHM system as it performs on a field test of the rover. In this scenario, the rover was executing various maneuvers, e.g., moving straight, turning at various speeds, and stopping. The current drawn from the batteries during these maneuvers is shown in Fig. 6.

As described in Section 3, the battery model is very accurate. As a result, the battery state can be confidently estimated, as shown in Fig. 5. The SOC estimate for a single battery is shown in Fig. 7. We plot here the apparent SOC, which is computed with respect to the charge available at the electrode surface. Due to the development of concentration gradients, apparent SOC may increase when the diffusion rate from the bulk to the surface layer exceeds the discharge rate of the battery, as observed in the figure. On larger time scales, SOC is seen to decrease steadily, and rover operation ceases once SOC reaches around $5 \%$.

The future power demands on the battery are unknown, especially in the driving scenario presented here, where an operator is deciding spontanteously where to drive the rover. If the

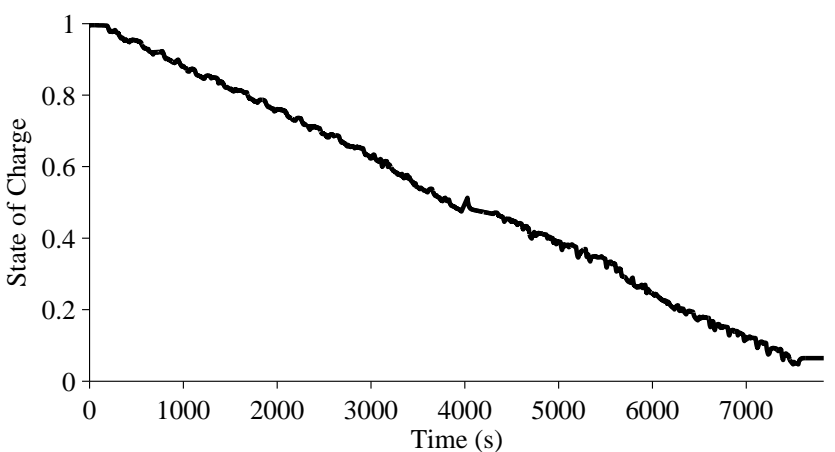

Figure 7. Estimated battery state of charge.

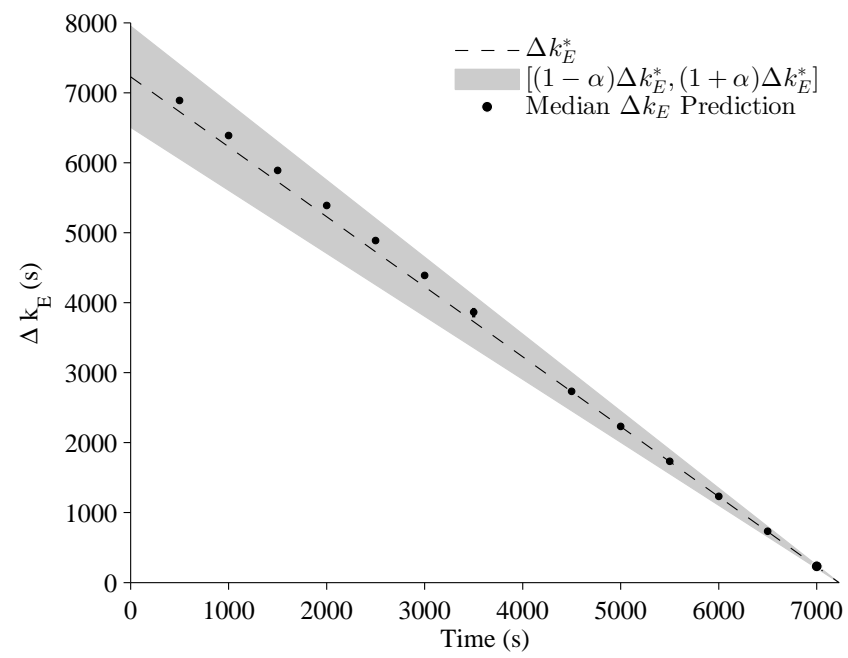

Figure 8. $\Delta k_{E}$ predictions with known future inputs.

future usage of the rover is known exactly, the BHM system should be able to obtain accurate predictions, since the model is accurate. Prediction results in this case are shown in Fig. 8, and the results are very accurate. There is some slight positive bias, which is due in part to sensor noise, because EOD is determined based on measured battery voltage, which, due to noise, will be earlier than actual EOD because the voltage threshold will be reached first due to noise. Overall, results are still very accurate, and have virtually no spread, since the estimation results had very little variance. Relative accuracy of the predictions averages to $98 \%$, with a spread of only $0.16 \%$ relative standard deviation. Accuracy can only be improved with a better model, and, given the model we have, represents the best possible performance. This serves as a baseline for the scenarios where the future inputs are not known.

If the future battery usage is unknown, we provide the operator with prediction results for three separate cases, best-, average-, and worst-case power demands. Prediction results in this case are shown in Fig. 9. Clearly, predictions assuming average-case usage are not accurate, as the actual power usage is less than the average case up until $4000 \mathrm{~s}$; after that the average power usage of the remainder of the scenario is greater than the average case. The average relative accuracy in this case is $83.3 \%$ based on the predictions corresponding to the average case. The predictions based on average usage still falls within the bounds set by the best- and worst-case usage. These results are with respect to continued usage, so 


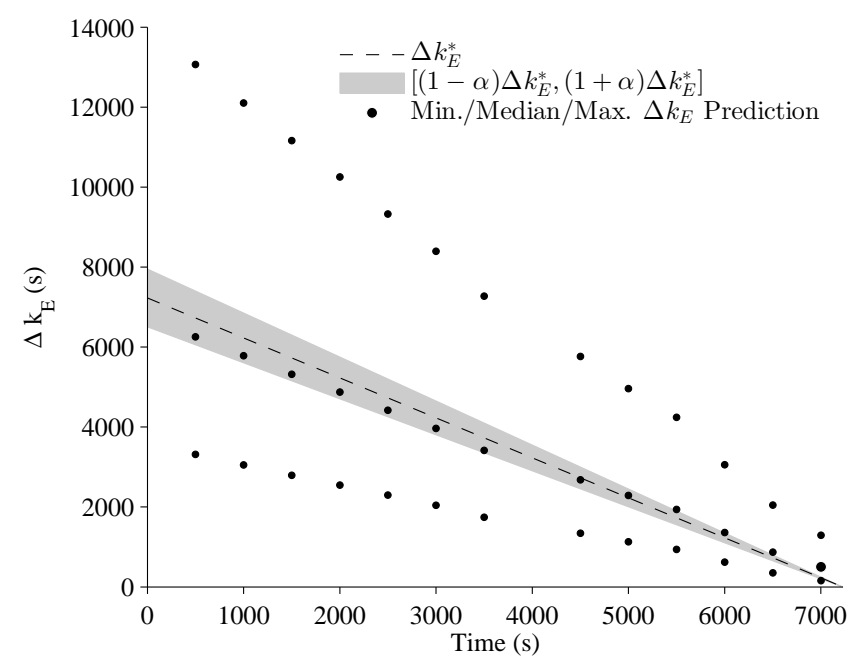

Figure 9. $\Delta k_{E}$ predictions at best-, average-, and worst-case usage.

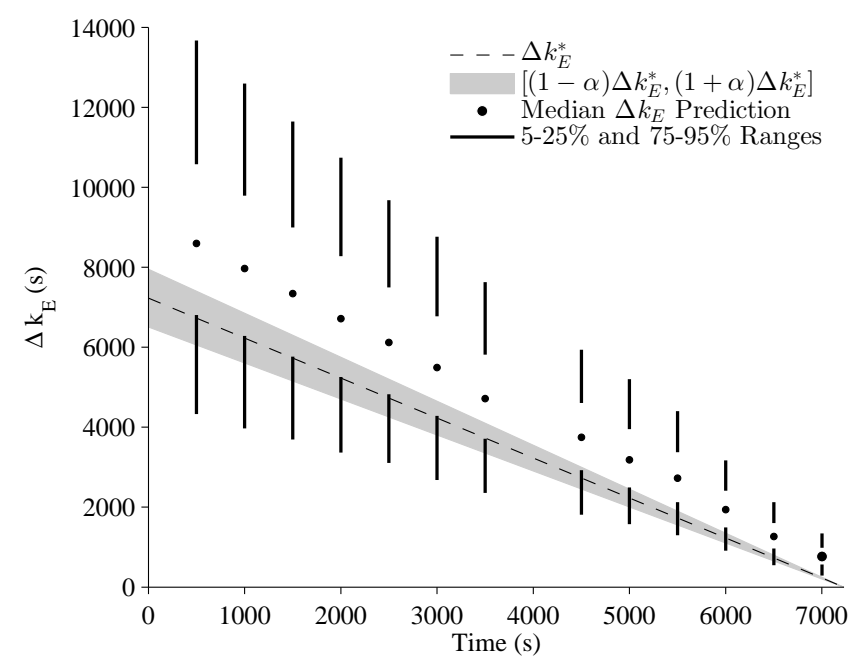

Figure 10. $\Delta k_{E}$ predictions for equivalent constant-loading.

the operator knows how much driving time is remaining, i.e., the predictions do not include time spent while the rover is stopped.

Using the equivalent constant-loading distribution, we obtain the predictions given in Fig. 10. Here, because the actual mean power drawn in this scenario is larger than in the collection of field experiments, the median $\Delta k_{E}$ predictions are larger than the true values. Relative accuracy computed using these values is only $63 \%$. Because of the large variety of scenarios in the field experiments, the spread is large, at $45 \%$ relative standard deviation. These numbers can be improved only if additional information is known about the future usage and that information is captured in the future input characterization, e.g., through the use of additional surrogate variables [11].

\section{Conclusions}

In this paper, we described a battery health monitoring framework for a rover. The prognostics framework was detailed, along with algorithms for state estimation and endof-discharge prediction. The provided battery model was validated with both laboratory and field test data. Validation of the BHM system was demonstrated using data from an actual field test of the rover.

Future work will include end-of-life prediction for the batteries, which must track the degradation of the battery health over multiple usage cycles. Initial results suggest that the battery model described here is applicable in this case [12]. In addition, when there is some knowledge available as to the future operation of the rover, e.g., a set of waypoints, or a large set of past driving scenarios, future inputs can be characterized in a more complex way than presented here, which can potentially lead to more accurate results with much less spread [11]. However, this depends on what kind of information is desired by the operator, as in many cases, predictions for best-, average-, and worst-case usage are sufficient.

\section{ACKNOWLEDgments}

This work was funded in part by the NASA System-wide Satefy Assurance Technologies (SSAT) project under the Aviation Safety (AvSafe) Program of the Aeronautics Research Mission Directorate (ARMD). The authors also acknowledge Adam Sweet, NASA Ames Research Center, for obtaining field test data.

\section{REFERENCES}

[1] S. Narasimhan, E. Balaban, M. Daigle, I. Roychoudhury, A. Sweet, J. Celaya, and K. Goebel, "Autonomous decision making for planetary rovers using diagnostic and prognostic information," in Proceedings of the 8th IFAC Symposium on Fault Detection, Supervision and Safety of Technical Processes, Aug. 2012, pp. 289-294.

[2] L. Tang, E. Hettler, B. Zhang, and J. Decastro, "A testbed for real-time autonomous vehicle PHM and contingency management applications," in Annual Conference of the Prognostics and Health Management Society 2011, Oct. 2011, pp. 56-66.

[3] B. Saha and K. Goebel, "Modeling Li-ion battery capacity depletion in a particle filtering framework," in Proceedings of the Annual Conference of the Prognostics and Health Management Society 2009, Sep. 2009.

[4] B. Saha, K. Goebel, S. Poll, and J. Christophersen, "An integrated approach to battery health monitoring using Bayesian regression and state estimation," in 2007 IEEE Autotestcon, 2007, pp. 646-653.

[5] — "Prognostics methods for battery health monitoring using a Bayesianframework," IEEE Transactions on Instrumentation and Measurement, vol. 58, no. 2, pp. 291-296, Feb. 2009.

[6] B. Saha, C. C. Quach, and K. Goebel, "Optimizing battery life for electric UAVs using a Bayesian framework," in Proceedings of the 2012 IEEE Aerospace Conference, Mar. 2012.

[7] C. Quach, B. Bole, E. Hogge, S. Vazquez, M. Daigle, J. Celaya, A. Weber, and K. Goebel, "Battery charge depletion prediction on an electric aircraft," in Annual Conference of the Prognostics and Health Management Society 2013, Oct. 2013, pp. 503-512.

[8] J. Oliva, C. Weihrauch, and T. Bertram, "A model- 
based approach for predicting the remaining driving range in electric vehicles," in Annual Conference of the Prognostics and Health Management Society 2013, Oct. 2013, pp. 438-448.

[9] M. Daigle, A. Saxena, and K. Goebel, "An efficient deterministic approach to model-based prediction uncertainty estimation," in Annual Conference of the Prognostics and Health Management Society, Sep. 2012, pp. 326-335.

[10] M. Daigle, B. Saha, and K. Goebel, "A comparison of filter-based approaches for model-based prognostics," in Proceedings of the 2012 IEEE Aerospace Conference, Mar. 2012.

[11] M. Daigle and S. Sankararaman, "Advanced methods for determining prediction uncertainty in model-based prognostics with application to planetary rovers," in Annual Conference of the Prognostics and Health Management Society 2013, Oct. 2013, pp. 262-274.

[12] M. Daigle and C. Kulkarni, "Electrochemistry-based battery modeling for prognostics," in Annual Conference of the Prognostics and Health Management Society 2013, Oct. 2013, pp. 249-261.

[13] E. Balaban, S. Narasimhan, M. Daigle, I. Roychoudhury, A. Sweet, C. Bond, and G. Gorospe, "Development of a mobile robot test platform and methods for validation of prognostics-enabled decision making algorithms," International Journal of Prognostics and Health Management, vol. 4, no. 1, May 2013.

[14] S. Sankararaman, M. Daigle, A. Saxena, and K. Goebel, "Analytical algorithms to quantify the uncertainty in remaining useful life prediction," in Proceedings of the 2013 IEEE Aerospace Conference, Mar. 2013.

[15] M. Daigle and K. Goebel, "Model-based prognostics with concurrent damage progression processes," IEEE Transactions on Systems, Man, and Cybernetics: Systems, vol. 43, no. 4, pp. 535-546, May 2013.

[16] C. D. Rahn and C.-Y. Wang, Battery Systems Engineering. Wiley, 2013.

[17] D. K. Karthikeyan, G. Sikha, and R. E. White, "Thermodynamic model development for lithium intercalation electrodes," Journal of Power Sources, vol. 185, no. 2, pp. 1398-1407, 2008.

[18] S. J. Julier and J. K. Uhlmann, "A new extension of the Kalman filter to nonlinear systems," in Proceedings of the 11th International Symposium on Aerospace/Defense Sensing, Simulation and Controls, 1997, pp. 182-193.

[19] —-, "Unscented filtering and nonlinear estimation," Proceedings of the IEEE, vol. 92, no. 3, pp. 401-422, Mar 2004.
[20] M. S. Arulampalam, S. Maskell, N. Gordon, and T. Clapp, "A tutorial on particle filters for online nonlinear/non-Gaussian Bayesian tracking," IEEE Transactions on Signal Processing, vol. 50, no. 2, pp. 174-188, 2002.

\section{BIOGRAPHY}

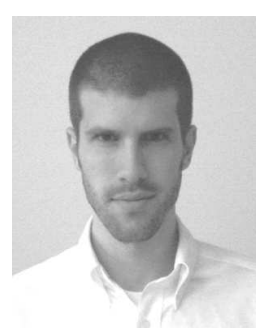

Matthew Daigle received the B.S. degree in Computer Science and Computer and Systems Engineering from Rensselaer Polytechnic Institute, Troy, NY, in 2004, and the M.S. and Ph.D. degrees in Computer Science from Vanderbilt University, Nashville, TN, in 2006 and 2008, respectively. From September 2004 to May 2008, he was a Graduate Research Assistant with the Institute for Software Integrated Systems and Department of Electrical Engineering and Computer Science, Vanderbilt University, Nashville, TN. From June 2008 to December 2011, he was an Associate Scientist with the University of California, Santa Cruz, at NASA Ames Research Center. Since January 2012, he has been with NASA Ames Research Center as a Research Computer Scientist. His current research interests include physicsbased modeling, model-based diagnosis and prognosis, simulation, and hybrid systems. Dr. Daigle is a member of the Prognostics and Health Management Society and the IEEE.

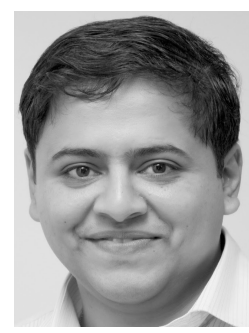

Chetan S. Kulkarni received the B.E. (Bachelor of Engineering) degree in Electronics and Electrical Engineering from University of Pune, India in 2002 and the M.S. and Ph.D. degrees in Electrical Engineering from Vanderbilt University, Nashville, TN, in 2009 and 2013, respectively. In 2002 he joined Honeywell Automation India Limited (HAIL) as a Project Engineer. From May 2006 to August 2007 he was a Research Fellow at the Indian Institute of Technology (IIT) Bombay with the Department of Electrical Engineering. From Aug 2007 to Dec 2012, he was a Graduate Research Assistant with the Institute for Software Integrated Systems and Department of Electrical Engineering and Computer Science, Vanderbilt University, Nashville, TN. Since January 2013 he has been a Research Engineer II with SGT Inc. at the Prognostics Center of Excellence, NASA Ames Research Center. His current research interests include physics-based modeling, model-based diagnosis and prognosis focused towards electrical and electronic devices and systems. Dr. Kulkarni is a member of the Prognostics and Health Management (PHM) Society, AIAA and the IEEE. 